\title{
Percepción de pobreza y de apoyo social en adolescentes paraguayos
}

\section{Perception of poverty and social support in paraguayan adolescents}

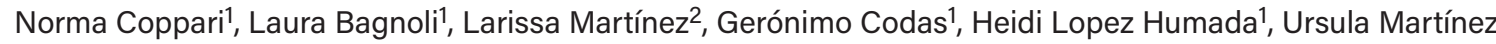 \\ Cañete ${ }^{1}$, Maureen Montanía ${ }^{2}$ \\ 'Universidad Católica de Asunción, CONACYT Asunción, Paraguay \\ ²Universidad Católica de Asunción, Asunción, Paraguay \\ (Rec.: febrero de 2018 - abril de 2018)
}

\begin{abstract}
Resumen
Se analiza la relación entre los niveles de percepción de pobreza y de apoyo social percibido en 1334 adolescentes paraguayos, 609 varones y 725 mujeres, de entre 12 y 18 años $(M=14.99 ; D E=1.66)$. Los resultados muestran variables relacionadas. A mayor percepción de pobreza menor percepción de apoyo social. El análisis de regresión permite predecir una variable a partir de la otra. No se hallaron correlaciones entre edad y las variables estudiadas, por otro lado, se observaron diferencias significativas en las escalas de apoyo social y en la percepción de pobreza frente a los rangos etarios, puntuando más alto los adolescentes de 16 a 18 años. Se encontraron diferencias significativas en cuanto al sexo, siendo los hombres quienes mayor puntúan en las escalas de apoyo social de amigos y otros. No se hallaron diferencias significativas en cuanto al apoyo social familiar y edad. Se rescata la importancia del diseño de políticas públicas que fortalezcan los lazos de ayuda dentro de comunidades excluidas socialmente, en tanto estos resultados muestran el apoyo social percibido por los adolescentes como un recurso para sobrellevar situaciones de vulnerabilidad y pobreza.
\end{abstract}

Palabras clave: Adolescentes, Apoyo social, edad, percepción, pobreza, sexo.

\begin{abstract}
This paper analyzes the relationship between the levels of perception of poverty and perceived social support in 1334 Paraguayan adolescents, 609 men and 725 women, aged between 12 and 18 years old $(M=14.99 ; D E=1.66)$. The results show that the variables are related: the higher the perception of poverty, the lower the perceived social support. The regression analysis allows us to predict one variable from the value of another. There were no correlations between age and the studied variables, but there were significant differences in the scales of perception of poverty and perceived social support compared to age ranges, where adolescents aged 16 to 18 got higher scores. Moreover, there were significant differences in terms of sex, where men have higher scores in scales of social support of friends and others. There were no significant differences related to family social support and age. It is relevant to point out the importance of designing public policies that strengthen the aid within socially excluded communities, since the results show that the social support is a resource perceived by adolescents to cope with situations of vulnerability and poverty.
\end{abstract}

Keywords: Adolescents, social support, age, perception, poverty, sex

Correspondencia: Norma Coppari. Universidad Católica de Asunción, Asunción, Paraguay. Mail: norma@tigo.com.py. Francis Morices 590. Asunción, Paraguay. Tel: +595981 727886. Dr. Norma Coppari (MS., ME). Investigadora nivel II, PRONII-CONACYT, Paraguay. Autora e investigadora principal del proyecto 14-INV-424. Financiado por PROCIENCIA, CONACYT, Paraguay. Institución beneficiada: Universidad Católica de Asunción. 


\section{Introducción}

La pobreza y la desigualdad, son realidades que afectan a muchas personas, especialmente en Latinoamérica (Boltvinik \& Damián, 2016; Vakis, Rigolini, \& Lucchetti, 2015), asociándose ambas con un mayor riesgo de mala salud (De la Caridad, 2013; Fresno, Spencer, Leiva \& Gallardo, 2011; Marmot, 2015; Office for National Statistics, 2014). Existe evidencia además, de la relación directa e indirecta entre factores socioeconómicos como el Estatus Socioeconómico (ESE) y el desarrollo de trastornos mentales en la adolescencia (Conger \& Conger, 2002).

Teniendo en cuenta una perspectiva objetiva, la pobreza involucra juicios normativos sobre lo que constituye la pobreza y qué se necesita para salir de ella. Supone la carencia o insuficiencia de un atributo (monetario o no monetario) con relación a un umbral, por debajo del cual se hallará en pobreza (Casas \& Barichello, 2015). Por otro lado, la pobreza subjetiva o percepción de pobreza tiene en cuenta las preferencias de los individuos considerados "pobres" o el valor que le dan a los bienes y servicios, por lo tanto hace énfasis en la utilidad individual de las personas (Aguado \& Osorio, 2006). Esta percepción se asocia con las privaciones materiales para cubrir necesidades básicas como alimentación y vivienda, y otras menos imperiosas como el ocio, pero todas necesarias para el bienestar emocional (Barcelata \& Márquez-Caraveo, 2015).

En estudios con poblaciones en condiciones de pobreza, se identificaron dos grupos de factores protectores: internos, entre los cuales destacan el temperamento y la personalidad con tendencia a buscar apoyo; y externos, de índole familiar, tales como la cohesión y la flexibilidad. La presencia de una fuerte relación con una persona significativa se menciona como otro factor protector en ambientes de deprivación social y condiciones de pobreza (Barrera \& Prelow, 2000; Rutter, 2002; Werner \& Smith, 1992).

En América Latina, se señala que la pobreza tiene "cara de niño" (UNICEF, 2005). En efecto, la pobreza infantil se asocia con carencias que producen daños biológicos permanentes, además de comprometer su desarrollo psicológico y social (Centro de Investigación Aplicada, 2017).

En el caso de Paraguay, hasta 2015 las estimaciones de pobreza estaban basadas en las líneas de pobreza definidas a partir de la Encuesta Integrada de Hogares realizada en 1997/98, sin embargo, dichas líneas de pobreza, ancladas en una estructura de consumo que data de hace dos décadas, quedaron obsoletas. La revisión detallada implicó la elaboración de una nueva serie de cifras que incluye el periodo 1997 al 2016 (Dirección General de Estadísticas, Encuestas y Censos, 2016), considerándose las Encuestas de Hogares y el método de la Línea de pobreza, para estimar la proporción de población en situación de pobreza y pobreza extrema.

En este sentido, se define como "población pobre" a aquel conjunto de personas residentes en hogares cuyo nivel de bienestar (medido a través del ingreso) es inferior al costo de una Canasta Básica de Consumo constituida por el conjunto de bienes y servicios que satisfacen ciertos requerimientos mínimos, tanto alimentarios como no alimentarios. El costo de esta Canasta Básica de Consumo se denomina Línea de pobreza Total (LPT) (DGEEC, 2016).
La Línea de Pobreza Total (LPT) se construye estimando primero el costo de una canasta básica de alimentos cuyo contenido calórico y proteico satisfaga los requerimientos nutricionales de la población y luego, se le añade el costo de la canasta básica no alimentaria, compuesta por otros bienes y servicios esenciales, relacionados con la vivienda, vestido, educación, entre otros. El costo mensual por persona de la canasta básica de alimentos se denomina Línea de Pobreza Extrema ( $L P E)$. Se define como población en pobreza extrema al conjunto de personas que viven en hogares cuyos ingresos per cápita son inferiores al costo de una Canasta Básica de Alimentos (DGEEC, 2016).

La población paraguaya considerada en situación de pobreza representa $28,86 \%$ del total de habitantes del país, lo que significa que alrededor de 1 millón 900 mil personas residen en hogares cuyos ingresos per cápita son inferiores al costo de una canasta básica de consumo, estimado para dicho año. En el área rural, la pobreza total afecta en el mismo año al 39,72\% de su población, mientras que el área urbana presenta una menor proporción de habitantes viviendo en condiciones de pobreza (21,94\%). En términos absolutos, la mayor cantidad de población pobre se encuentra en el área rural, albergando a más del millón de personas en tal situación, en tanto en el área urbana el número de pobres suma 900 mil personas. En consecuencia, en el ámbito rural la pobreza es más extendida tanto en incidencia como en cantidad absoluta (DGEEC, 2016).

Por otra parte, la población en extrema pobreza o indigente (aquella cuyo ingreso mensual per cápita no logra cubrir el costo de una canasta mínima de consumo alimentario), llega a 387 mil personas aproximadamente, siendo mayor su proporción en el área rural $(12,17 \%$ de sus habitantes, en contraposición a un 1,63\% de la población urbana). Igualmente, el mayor número de pobres extremos se encuentra en el área rural, albergando a aproximadamente 320 mil de ellos (cerca del $83 \%$ de los pobres extremos). Así, el área rural es la más afectada por la indigencia tanto en incidencia (porcentaje) como en valores absolutos (cantidad de personas) (Encuesta Permanente de Hogares, 2016).

A los datos anteriores se suma que en Paraguay, de 1.500.000 menores de 10 años, el $40 \%$ se encuentra en situación de pobreza, teniendo que sobrevivir en sus hogares con un ingreso inferior a 21.000 guaraníes por día (alrededor de 4 USD), y el $9 \%$ vive en pobreza extrema o indigencia. Una gran proporción de niños y niñas en situación de pobreza carece de oportunidades para lograr el bienestar actual y futuro, ya que no poseen acceso a niveles mínimos de nutrición, educación, recreación, salud y vivienda (CIA, 2017). La pobreza infantil supone un alto costo social en términos de capital humano y de integración, y si se aspira a romper este ciclo intergeneracional de pobreza es imperante empezar con los más jóvenes (UNICEF, 2005).

El apoyo social percibido se refiere a la percepción que tienen las personas de la disponibilidad de los recursos sociales (Cohen \& Willis, 1985). Kaniasty y Norris (2001) lo definen como aquellas interacciones sociales que proporcionan a los individuos asistencia real o una red fácilmente disponible en tiempos de necesitad.

Esta variable ha sido asociada con mejor salud (Matud, Correa \& García, 2017; Bowen et al., 2013; Hamui-Sutton, Ponce-Ro- 
sas, Irigoyen-Coria \& Halabe-Cherem, 2009) y como un factor psicosocial asociado al bienestar en la adolescencia (Chu, Saucier \& Hafner, 2010; Rodríguez-Fernández, Ramos-Díaz, Ros, Fernández-Zabala \& Revuelta, 2016). Más específicamente, también se le tiene en cuenta para mejorar las estrategias de adherencia al tratamiento en pacientes que sufren enfermedades crónicas (Martos \& Pozo, 2011), tales como diabetes (Teherán, Álvarez, Muñoz, Barrera \& Cadavid, 2017), adicciones (Ballester, 2003; Bond, Kaskutas \& Weisner, 2003; Montesinos, Lloret, Segura \& Aracil, 2016) o conductas delictivas (Bustamante, Álvarez, Herrera, \& Pérez-Luco, 2016).

Distintos autores señalan la importancia que exista una red de apoyo social durante la adolescencia, especialmente la relacionada a la familia (Azzollini, Bail, Vera \& Victoria, 2012; Barcelata, Granados \& Ramírez, 2013). De igual manera, la percepción que los jóvenes tengan respecto del apoyo con que cuentan (su disponibilidad, accesibilidad, fortalezas y deficiencias), constituyen elementos centrales al intentar reflexionar sobre los medios para favorecer el desarrollo en esta etapa de la vida (Chavarría \& Barra, 2014; Méndez \& Barra, 2008; Hickey, Fitzgerald \& Dooley, 2017; Ramos-Díaz, Rodríguez-Fernández, Fernández-Zabala \& Zuazagoitia, 2016).

En cuanto a la relación entre percepción de pobreza y apoyo social se reporta que existe una relación entre ambas variables, teniendo en cuenta una subdivisión de los niveles socioeconómicos: pobres extremos, pobres moderados y no pobres (Palomar \& Cienfuegos, 2007), siendo los pobres los que perciben un menor apoyo social percibido y estructural que la clase media (Rodríguez, 2010). También este vínculo se observa en el caso de personas de tercera edad (Salazar, Pereira, Silveira \& Lost, 2013), y en estudios con otros grupos, se resalta la importancia del apoyo social percibido como factor que fortalece a la comunidad pobre (Barrón \& Sánchez, 2001; Martín, Fajardo, Gutiérrez \& Palma, 2007; Gracia \& Herrero, 2006).

En cuanto a las variables sociodemográficas que se relacionan con estos dos constructos (percepción de pobreza y de apoyo social), se reporta que la percepción de apoyo conforma uno de los principales recursos del adolescente para hacer frente a cambios y situaciones nuevas o conflictivas (Musitu \& Cava, 2003), tales como la pobreza (UNICEF, 2005). Cabe señalar que los adolescentes que viven con presiones económicas son más propensos a experimentar sucesos de vida adversos (Barcelata \& Lucio, 2012). La percepción del apoyo social en ocasiones depende de la edad y el sexo de los mismos (Barcelata et al., 2013; Barcelata, Coppari \& Márquez-Caraveo, 2014; Hickey et al, 2017: Musitu \& Cava, 2003), y en otras, se muestra independiente de variables culturales, de sexo y edad, como se demostró en un estudio transcultural de adolescentes paraguayos y mexicanos (Barcelata et al., 2014, Rodríguez, 2010).

Dentro de este contexto local radica la necesidad de investigar acerca de la situación de pobreza, específicamente en adolescentes paraguayos, y cómo el apoyo social percibido podría estar asociado a ella. Asimismo, como investigación aplicada a un problema social y financiada por el Estado, las evidencias obtenidas serán capitalizadas en políticas públicas que promuevan formas de empoderamiento, educación y prevención de riesgos, que consideren las formas de apoyo social como redes protectoras del grupo adolescente de interés.

De esta manera, los objetivos del estudio fueron: (1) identifi- car la percepción y el nivel de relación entre el apoyo social, en cada una de sus escalas, y la percepción de pobreza en adolescentes, varones y mujeres, de entre 13 y 18 años de la ciudad de Asunción, (2) describir la distribución de la muestra por sexo y edad, y la percepción de apoyo social en cada uno de sus factores, (3) determinar el coeficiente de correlación entre los puntajes de apoyo social percibido, por escalas, y total, y la percepción de pobreza en adolescentes paraguayos, conforme edad y sexo.

\section{Método}

El diseño utilizado fue no experimental, transversal y correlacional (McGuigan, 1996).

\section{Participantes}

El muestreo utilizado fue intencionado y autoselectivo. Participaron 1.334 adolescentes de una institución pública de Educación Escolar Básica y Educación Media de la Ciudad de Asunción. El rango de edad fue de entre 13 y 18 años $(M=14.99$; $D E=1.66)$. En cuanto a distribución por sexo, 609 de los participantes son hombres y 725 son mujeres, todos ellos pertenecientes a un nivel socioeconómico bajo.

Se observa (Tabla 1) una mayor cantidad de estudiantes en el rango de 12 a 15 años en comparación a los estudiantes de 16 a 18 años, siendo el promedio de la edad de los participantes, $M=14,99$ y $D E=1.66$. Hubo preponderancia del sexo femenino en la muestra.

Tabla 1. Datos sociodemográficos: Edad y Sexo de los participantes

\begin{tabular}{ccc}
\hline Edad & Frecuencia & Porcentaje \\
\hline 12 a 15 años & 770 & 57.7 \\
16 a 18 años & 564 & 42.3 \\
Sexo & & \\
Masculino & 609 & 45.7 \\
Femenino & 725 & 54.3 \\
\hline
\end{tabular}

En cuanto a las características sociodemográficas de los progenitores de los estudiantes, encontramos que respecto a su escolaridad se encontró un porcentaje similar tanto para padres como madres (27\%) que corresponde al nivel secundaria/escuela técnica, respecto a su ocupación, se observa un mayor porcentaje en empleado/oficinista (34,5\%) en el caso de los padres, y ama de casa (39,8\%) en la ocupación de las madres. La mayoría indicó el ingreso mensual aproximado de la familia como más de $\$ 9.000$ (39\%), también indicaron a ambos padres como proveedores en la familia $(38,8 \%)$.

\section{Instrumentos}

Se aplicó la Cédula Sociodemográfica para el Adolescente y su Familia (Barcelata, 2013). Es un instrumento mixto integrado por 26 reactivos, categóricos y de opción múltiple. Evalúa la estructura familiar, por ejemplo: vivo con mi padre (sí, no); nivel educativo (primaria, secundaria, bachillerato); ocupación de los padres (obrero, oficinista, comerciante, entre otros), y estado civil de los padres (p.ej. casado, divorciado, unión libre); condiciones de infraestructura de la vivienda y bienes materia- 
les (p.ej. cuarto, departamento, casa sola, etc.; zona de residencia o van a la escuela). La fiabilidad de este instrumento con $\alpha$ de Kuder-Richardson $=0.65$; y un $\alpha$ nominal $=0.69$ ). El instrumento permite establecer el nivel socioeconómico a partir de las condiciones de infraestructura y bienes (Rosenblüth, 2006)

Por otra parte, se implementó la Escala de Apreciación de Apoyo Social -EAAS de Martínez (2004). Adaptada para población mexicana de la Social Support Appraisal Scale de Cobb (1976), se empleó para evaluar el apoyo social percibido. Consta de 23 reactivos con opciones de respuesta tipo Likert, que va de 1 a 4 puntos, donde 1= es "muy de acuerdo" y 4= "muy en desacuerdo", organizados en tres factores: 8 reactivos para familia, 7 para amigos, y 8 para otros. En su conjunto evalúa apreciación de apoyo social, por medio del cual el adolescente puede valorar su percepción, centrado en su propia experiencia y en la percepción de disponibilidades de conductas de apoyo por parte de su familia, amigos y demás personas dentro de la sociedad en la cual se desenvuelve (Martínez, 2004). Se reportan alfas de Cronbach de .80 y .90 en su versión original, así como índices de 0.80 y de 0.77 en la versión adaptada y en otros estudios (Barcelata et al., 2013).

\section{Procedimiento}

Teniendo en cuenta el código de Ética de American Psychological Association (2013) en todo el desarrollo de la investigación, se administraron consentimientos informados firmados por los padres y adolescentes, asegurando el protocolo ético de la participación voluntaria, anónima y confidencial de los particpantes. De igual manera, la aplicación contó con la colaboración de investigadores auxiliares entrenados. No se dio un incentivo monetario, sino que se motivó a los participantes destacando la importancia de representar una muestra paraguaya, para generar datos sobre la adolescencia en el país. Los cuestionarios fueron aplicados en formato electrónico, y en formato de papel y lápiz dependiendo de los recursos de los participantes.

- En formato electrónico, se diseñó una encuesta online conteniendo todos los instrumentos del estudio. Se distribuyeron los cuestionarios vía redes sociales para que los participantes los completen libremente, siendo motivados por los investigadores, dentro de la institución educativa, para lograr una adhesión significativa.

- En formato papel y lápiz, se distribuyeron los cuestionarios en horario de clase, en el predio de la institución y en las mismas aulas, por medio de investigadores auxiliares debidamente entrenados para el efecto y con la supervisión de la investigadora titular.

Los cuestionarios completados online se corregían automáticamente a través de una matriz de Microsoft Excel. Los casos evaluados a través del formato papel y lápiz fueron corregidos de forma manual, cargados individualmente en la misma matriz de datos, y fidelizados por otros integrantes del grupo de investigación. La duración de esta actividad fue de 1 mes.

\section{Análisis de datos}

Los datos obtenidos se sometieron al software SPSS v. 15, analizando las variables sociodemográficas y los puntajes de los cuestionarios de acuerdo a medidas de centralización y dispersión, en un lapso de 2 semanas. Con el propósito de describir la muestra, se realizaron análisis descriptivos y comparativos de las variables sociodemográficas básicas, como edad, sexo de los adolescentes, ocupación y escolaridad de los padres, que se consideran marcadores socioeconómicos (Rosenblüth, 2006). Los análisis inferenciales utilizados fueron la correlación de Pearson y el análisis de regresión lineal, así como la prueba t y la U-Mann Whitney.

\section{Resultados}

Se observa (Tabla 2), una correlación alta e inversa entre los puntajes de la percepción de pobreza y del apoyo social percibido, siendo el valor del análisis de Pearson -0.69.

Tabla 2. Correlación entre Percepción de pobreza y Apoyo social

\begin{tabular}{lccc}
\hline & $\begin{array}{c}\text { Percepción de } \\
\text { Pobreza }\end{array}$ & Apoyo Social & Edad \\
\hline $\begin{array}{l}\text { Percepción de } \\
\text { pobreza } \\
\text { Apoyo Social }\end{array}$ & & $-0.69 * * *$ & -0.12 \\
\hline
\end{tabular}

Nota. ${ }^{*} p<.05 ;{ }^{*} p<.01 ;{ }^{* * *} p<.001$.

Estos resultados evidencian, en esta muestra de estudio, que a mayor percepción de pobreza, menor es la del apoyo social percibido por los adolescentes. Por otro lado, no se encontró correlación de la edad con la percepción de pobreza, ni con el apoyo social.

Como se aprecia (Tabla 3), es posible predecir los puntajes de apoyo social percibido de los adolescentes, a partir de los correspondientes a la percepción de pobreza, tomando en cuenta que por cada punto en esta última medida, habría un decremento de alrededor de 1,69 puntos en el cuestionario de apoyo social.

Tabla 3. Regresión de Apoyo social a partir de los puntajes de Percepción de pobreza

\begin{tabular}{ccc}
\hline B & B & $\begin{array}{c}\text { Intervalo de } \\
\text { confianza 95\% }\end{array}$ \\
\hline-1.69 & -0.69 & $(-1.78,-1.60)$ \\
\hline
\end{tabular}

Nota. B: Coeficiente no estandarizado; ß: Coeficiente estandarizado.

El coeficiente de correlación ntre la edad y las escalas de apoyo social (Tabla 4), que evalúan el apoyo social de la familia, de las amistades y el percibido de otros individuos, presenta valores muy bajos, por lo que no se puede indicar que exista correlación entre las variables. 
Tabla 4. Correlación entre las escalas de Apoyo social y Edad

\begin{tabular}{ll}
\hline Escalas de Apoyo social & Edad \\
\hline & $\mathrm{r}(\mathrm{p}$ valor $)$ \\
\hline Apoyo social en la Familia & 0.13 \\
& $(0.00)$ \\
\hline Apoyo social en la Amistad & 0.10 \\
& $(0.00)$ \\
\hline Apoyo social en otros individuos & 0.11 \\
& $(0.02)$ \\
\hline
\end{tabular}

Por otro lado, se puede apreciar que existen diferencias significativas entre las diferentes escalas de apoyo social percibido frente a la franja etaria de los adolescentes (Tabla 5).

Tabla 5. Escalas de Apoyo social y Percepción de pobreza frente a la edad

\begin{tabular}{|c|c|c|c|c|c|}
\hline Escalas & Edad & $\mathrm{N}$ & Media & $\mathrm{T}$ & $P$ \\
\hline \multirow{2}{*}{$\begin{array}{l}\text { Apoyo social } \\
\text { en la Familia }\end{array}$} & 12 a 15 años & 770 & 25.44 & \multirow{2}{*}{-3.51} & \multirow{2}{*}{0.00} \\
\hline & 16 a 18 años & 564 & 27.17 & & \\
\hline \multirow{2}{*}{$\begin{array}{l}\text { Apoyo social } \\
\text { en la Amistad }\end{array}$} & 12 a 15 años & 770 & 15.73 & \multirow{2}{*}{-3.16} & \multirow{2}{*}{0.00} \\
\hline & 16 a 18 años & 564 & 16.68 & & \\
\hline \multirow{2}{*}{$\begin{array}{l}\text { Apoyo social } \\
\text { en otros } \\
\text { individuos }\end{array}$} & 12 a 15 años & 770 & 21.27 & \multirow[b]{2}{*}{-3.64} & \multirow[b]{2}{*}{0.00} \\
\hline & 16 a 18 años & 564 & 22.53 & & \\
\hline \multirow{2}{*}{$\begin{array}{l}\text { Apoyo social } \\
\text { total }\end{array}$} & 12 a 15 años & 770 & 62.45 & \multirow{2}{*}{-3.68} & \multirow{2}{*}{0.00} \\
\hline & 16 a 18 años & 564 & 66.38 & & \\
\hline \multirow{2}{*}{$\begin{array}{l}\text { Percepción } \\
\text { de pobreza }\end{array}$} & 12 a 15 años & 770 & 25.54 & \multirow{2}{*}{4.13} & \multirow{2}{*}{0.00} \\
\hline & 16 a 18 años & 564 & 23.75 & & \\
\hline
\end{tabular}

A partir de estos resultados, se advierte que en la percepción del apoyo social de la familia, de las amistades, de otros individuos y del apoyo social total, los participantes de 16 a 18 años recibieron puntajes más elevados que aquellos entre 12 a 15 años. Así también, se hallan diferencias significativas al comparar los valores de la percepción de pobreza y la edad de los adolescentes, mostrando mayor puntaje los adolescentes de 12 a 15 años, siendo pequeño el tamaño del efecto de las diferencias $(d=0.23)$.

Finalmente, se puede notar (tal como se refleja en la Tabla 6 ), que existen diferencias significativas entre los puntajes de hombres y mujeres en las diferentes escalas de percepción de apoyo social, encontrándose que los valores de percepción del apoyo social total, de las amistades y de otros individuos, son superiores entre los varones. Por otro lado, en cuanto a la percepción de apoyo social de la familia, no hay diferencias significativas entre ambos sexos.
Tabla 6. Escalas de Apoyo social y Percepción de pobreza frente al sexo

\begin{tabular}{lccccc}
\hline \multicolumn{1}{c}{ Escalas } & sexo & $\mathrm{N}$ & Media & $\mathrm{t}$ & $\mathrm{P}$ \\
\hline $\begin{array}{l}\text { Apoyo social } \\
\text { en la Familia }\end{array}$ & Masculino & 609 & 26.45 & & \\
& Femenino & 725 & 25.94 & 1.05 & 0.29 \\
$\begin{array}{l}\text { Apoyo } \\
\text { social en la }\end{array}$ & Masculino & 609 & 16.64 & & \\
$\begin{array}{l}\text { Amistad } \\
\text { Apoyo social }\end{array}$ & Femenino & 725 & 15.71 & & 0.00 \\
$\begin{array}{l}\text { en otros } \\
\text { individuos }\end{array}$ & Femenino & 725 & 21.48 & & \\
\hline $\begin{array}{l}\text { Apoyo } \\
\text { social total }\end{array}$ & Masculino & 609 & 19.44 & & \\
\hline $\begin{array}{l}\text { Percepción } \\
\text { de pobreza }\end{array}$ & Masculino & 609 & 24.42 & & \\
\hline
\end{tabular}

Como en la comparación anterior, no hay diferencias significativas entre la percepción de pobreza y el sexo de los adolescentes.

\section{Discusión}

En cuanto a la relación entre los puntajes de apoyo social percibido, se observa que las variables de interés de este estudio están vinculadas. A mayor percepción de pobreza, menor es la percepción de apoyo social en adolescentes paraguayos. Los resultados permiten predecir una percepción a partir de la otra. Estos hallazgos suponen que al percibir un menor apoyo social por parte de los demás, los adolescentes podrían tener también una valoración negativa de los recursos con los que cuentan para afrontar su condición de pobreza. Estos resultados coinciden con otros de estudios similares, que hablan de la relación entre estas variables (Barrera \& Prelow, 2000; Barrón \& Sánchez, 2001; Palomar \& Cienfuegos, 2007; Rodríguez, 2010; Rutter, 2002; Salazar et al., 2013; Werner \& Smith, 1992), y del impacto que pueden tener en la mayor o menor vulnerabilidad de los adolescentes.

Con respecto a la percepción de apoyo social conforme edad, se hallaron diferencias significativas, con un efecto de tamaño pequeño en cuanto al rango etario que comprende los 12 a 15 años. En el apoyo social, los adolescentes de 16 a 18 años puntúan más alto que aquellos entre 12 y 15 años. Esto contradice el estudio de Ramírez (2015) pero concuerda con que las etapas de la adolescencia marcan una diferencia en los niveles de apoyo social percibido (Musitu \& Cava, 2003). Sin embargo, es notable que estos autores hayan encontrado una disminución en la percepción de apoyo de los padres conforme se avanza en la adolescencia, a diferencia de los hallazgos del presente estudio. Esta diferencia podría deberse a la creciente madurez de los adolescentes medios, en comparación a los tempranos. 
En cuanto a la percepción de apoyo social y el sexo de los participantes, existen diferencias significativas, coincidiendo con estudios donde el apoyo social es percibido de manera distinta según el sexo. No obstante, resultados anteriores presentan que las mujeres han percibido mayor apoyo social (Hickey et al., 2017), especialmente, de parte de la pareja y las amistades (Barcelata et al., 2013; Barcelata et al., 2014; Ramírez, 2015; Musitu \& Cava, 2003), en contraposición a lo que se encuentra en esta investigación. En efecto, los adolescentes masculinos de la muestra perciben mayor apoyo social de sus familias, pares y otros significativos, planteando una diferencia llamativa.

Hay que subrayar que estos resultados no coinciden con otros estudios que no evidencian diferencias importantes entre el sexo y el apoyo percibido de las familias (Barcelata et al., 2014; Musitu \& Cava, 2003; Rodríguez, 2010). Sin embargo, para Ramírez (2015) el hecho de que los hombres perciban más apoyo de sus familias, probablemente los lleve a sentirse menos presionados por su entorno, y más acompañados para sobrellevar sus problemas, en una perspectiva de género menos convencional.

Indagar sobre la percepción de apoyo social de los individuos, varones como mujeres, de cualquier edad, puede proporcionar una visión más integral de la presencia o ausencia de bienestar. Esto permitiría, a su vez, dilucidar un análisis menos reduccionista de la pobreza, más allá de alcanzar un indicador de nivel mínimo de ingresos o medios para subsistir. Así, esta se entendería como un fenómeno más complejo e influenciado por un acumulado de circunstancias particulares que dependen del contexto y el entorno donde se desarrolla la persona, y que por ende, involucra distintas dimensiones del bienestar (Aguado \& Osorio, 2006).

En cuanto al tipo de apoyo social percibido, ya sea familiar, de amigos o de otros, los resultados confirman estudios previos realizados en Latinoamérica (Azzollini et al., 2012; Barcelata et al., 2013; Barcelata et al., 2014; Hickey et al., 2017; Palomar \& Cienfuegos, 2007, Ramírez, 2013) y Sudamérica más específicamente (Sarriera J. C., Bedin, L., Abs, D., Calza, T., \& Casas, F. (2015), en donde la familia parece constituir la primera red a la que se acude para sentirse protegidos y acompañados. Este hallazgo supone un aspecto positivo para la muestra estudiada, ya que el apoyo familiar se relaciona con un autoconcepto positivo en los adolescentes y su nivel de implicación escolar (Ramos et al., 2016), altos niveles de resiliencia (Rodríguez-Fernández et al., 2016; Rodríguez-Fernández, Ramos-Díaz, Ros \& Zuazagoitia, 2018)) y satisfacción vital (Chavarría \& Barra, 2014; Sarriera, et al., 2015), todos aspectos relacionados con el funcionamiento adaptativo.

Por otro lado, que el apoyo social de la categoría otros (en donde se ubican las instituciones públicas entre otros servicios) se encuentre en último lugar, apunta a que los participantes del estudio perciben un apoyo casi inexistente por parte de estas entidades, lo que podría potenciar ese sentimiento de abandono y mayor vulnerabilidad. Cuando se percibe pobreza, también la percepción de apoyo de las instituciones es menor, y la resolución de los problemas de salud resulta más difícil (Hamui-Sutton et al., 2009).

Es posible que la población que percibe tener un nivel socioeconómico bajo deposite muy poca confianza en la sociedad e instituciones como fuentes de ayuda, resultando en sentimientos de conformismo e incluso resentimiento (Rodríguez, 2010). Este conformismo podría repercutir en el nivel de apoyo que creen recibir de los mismos, reforzando el sentimiento de inequidad biológica y psicosocial impuesta de manera temprana (Barcelata \& Márquez-Caraveo, 2015).

Las estructuras sociales actúan como un contexto que potencia o disminuye los factores de riesgo biopsicosocial (Méndez \& Barra, 2008), por lo que es posible que estos individuos consideren que tienen muy pocos recursos para subsistir, debido al escaso apoyo social que perciben por parte de las instituciones sociales de su comunidad.

Estos resultados hacen pensar en que el apoyo social percibido de manera adecuada, puede considerarse como una condición -no única pero sí necesaria- para la adaptación del individuo a las exigencias medioambientales (Martín et al, 2007; Palomar \& Cienfuegos, 2007), como en este caso, la situación de pobreza percibida.

El estudio pretende ser una aproximación a la percepción de adolescentes de Asunción, sobre el apoyo recibido por parte de su entorno más cercano, como lo son sus familiares y amigos. De esta manera contribuye a identificar posibles riesgos para la salud mental de los mismos, ya que el apoyo social es considerado como una función relacionada con el funcionamiento psicológico de los individuos.

A partir de lo encontrado, se sugiere a los profesionales de salud mental fortalecer el apoyo familiar y social, favoreciendo por ejemplo, la escucha familiar, la pertenencia a la comunidad y el sentido de cohesión, puesto que la familia, los amigos y la propia comunidad, son vistos como recursos informales disponibles que garantizan la continuidad de los servicios profesionales en salud (Fernández, 2005).

Asimismo, desde una perspectiva macro, urgen importantes reformas en el diseño de políticas públicas que busquen fortalecer los lazos de ayuda y credibilidad dentro de comunidades excluidas socialmente, ya que los datos resaltan la importancia del apoyo social como recurso para sobrellevar y afrontar la situación de pobreza. Cabe considerar, que las condiciones en las que se encuentra la comunidad de residencia influyen en la percepción de apoyo de sus habitantes (Gracia \& Herrero, 2006).

Iniciativas como el Programa de Talleres "Adolescencia Prevenida" (Coppari, 2013), de prevención del riego y promoción de la resiliencia, articulan alternativas de solución de problemas, formando como monitores a adolescentes, padres, maestros y otros miembros de la comunidad, empoderando en las escuelas a estos actores, y generando un efecto multiplicador de los beneficios a mediano y largo plazo. Por esta razón, programas de este tipo deberían ser incluidos en las políticas públicas de salud y educación, con un enfoque positivo, comunitario y de continuidad de los insumos, para amortiguar y superar las consecuencias negativas de la pobreza.

Las limitaciones del estudio pueden relacionarse con la falta de un muestreo probabilístico y el alcance a otras zonas más rurales del Paraguay, a fin de tener un panorama más completo de la situación de pobreza y apoyo. Otra limitación se refiere a un posible sesgo debido a la doble modalidad 
de administración de cuestionarios, teniendo en cuenta que esto podría influir en las respuestas de los participantes. Así también, podrían sugerirse estudios que tengan en cuenta variables psicológicas que influyan en el nivel del apoyo social percibido, como pueden ser la autoestima, el locus de control, entre otros.

\section{Referencias}

American Psychological Association (APA) (2013). Ethical Principles of Psychologists and Code of Conduct. Recuperado de http://www.apa.org/ ethics/code/ index.aspx

Aguado Q., L. F. \& Osorio M., A. M. (2006). Percepción subjetiva de los pobres: Una alternativa a la medición de la pobreza. Reflexión Política, 8(15), 26-40. Recuperado de http://www.redalyc.org/articulo. oa? id $=11001503$.

Azzollini S., Bail, P., Vera V. \& Victoria, A. (2012). El apoyo social y el autocuidado en diabetes tipo 2. Anuario de Investigaciones, 19 (1):109-13. Recuperado el 27/07/17 de script=sci_arttext\&pi$\mathrm{d}=$ S1851-16862012000100010\&lng=es\&tlng=es

Ballester, R. (2003). Eficacia terapéutica de un programa de intervención grupal cognitivo-comportamental para mejorar la adherencia al tratamiento y el estado emocional de pacientes con infección por VIH/ SIDA. Psicothema, 15, 517-523.

Boltvinik, J., \& Damián, A. (2016). Pobreza creciente y estructuras sociales cada vez más desiguales en México. Una visión integrada y crítica. Acta Sociológica, (70), 271-296. DOI:10.1016/j.acso.2017.01.012

Barcelata, B. (2013). Cédula sociodemográfica del adolescente y su familia (Versión para investigación). CDMX, México: FES Zaragoza, UNAM.

Barcelata, B., Coppari, N. \& Marquez-Caraveo, E. (2014). Gender and age differences in coping: A comparison between Mexican and Paraguayan adolescents. Stress and Anxiety, 249-260.

Barcelata, B., Granados, A. \& Ramírez, A. (2013). Correlatos entre funcionamiento familiar y apoyo social percibido en escolares en riesgo psicosocial. Revista Mexicana de Orientación Educativa, 10 (24), 65-70.

Barcelata, B. \& Lucio, E. (2012). Fuentes de estrés y su influencia en la adaptación psicológica en jóvenes con adversidad económica. En-Claves del pensamiento, 6(2), 31-48.

Barcelata, B. \& Márquez-Caraveo, M. E. (2015). Riesgo, pobreza y salud mental del adolescente. En B. Barcelata, Adolescentes en riesgo. Una mirada a partir de la resiliencia (pp. 37 - 61) México, CDMX: Manual Moderno.

Barrera, M. \& Prelow, H. (2000). Interventions to promote social support in children and adolescents. En D. Cicchetti, J. Rappaport, I. Sandler y R. Weissberg (Eds.), The promotion of wellness in children and adolescents. (pp. 309-340). Washington, DC, USA: Child Welfare League Association Press.

Barrón L.D.R., A.B. \& Sánchez M., E. (2001). Estructura social, apoyo social y salud mental. Psicothema, 13(1), 1723. Recuperado de https://www.unioviedo.es/reunido/index.php/PST/article/view/7841

Bond, J., Kaskutas, L. A. \& Weisner, C. (2003). The persistence influence of social networks and Alcoholics
Anonymous on abstinence. Journal on Studies on Alcohol and Drugs, 64, 579-588. https://doi. org/10.15288/jsa.2003.64.579

Bowen, K. S., Birmingham, W., Uchino, B. N., Carlisle, M., Smith, T. W., \& Light, K. C. (2013). Specific dimensions of perceived support and ambulatory blood pressure: Which support functions appear most beneficial and for whom? International Journal of Psychophisiology, 88(3), 317-324. http://dx.doi.or$\mathrm{g} / 10.1016 / \mathrm{j}$.ijpsycho.2012.03.004

Bustamante, Y. Álvarez, L., Herrera, E. \& Pérez-Luco, R. (2016). Apoyo social percibido y su influencia en el desistimiento delictivo: Evaluación del rol institucional. PSICOPERSPECTIVAS, 15(1), 132 - 144. Recuperado de https://search.proquest.com/openview/ d3da443d2213b24d10fe4901c011ba98/1?pq-origsite $=$ gscholar $\& \mathrm{cbl}=105527$

Casas, J. \& Barichello, R. (2015). Hacia una noción sobre la pobreza. Apuntes del Cenes, 34(59), 39-62. https:// doi.org/10.19053/22565779.2784

Sarriera, J. C., Bedin, L., Abs, D., Calza, T., \& Casas, F. (2015). Relationship between social support, life satisfaction and subjective well-being in Brazilian adolescents. Universitas Psychologica, 14(2), 459-474. DOI: http:// dx.doi.org.10.11144/Javeriana.upsy14-2. rbss

Centro de investigación aplicada (CIA) - Observatorio económico facultad de ciencias contables, administrativas y económicas (2017). La infantilización de la pobreza: el $40 \%$ de los niños vive en hogares con un ingreso per cápita inferior a 21 mil guaraníes por día. Recuperado el 27/07/2017 de http://www. universidadcatolica.edu.py/assets/documentos/ ucdoc_1500308619.pdf

Chavarría, M. P., \& Barra, E. (2014). Satisfacción Vital en Adolescentes: Relación con la Autoeficacia y el Apoyo Social Percibido. Terapia psicológica, 32(1), 41-46. https://dx.doi.org/10.4067/S071848082014000100004

Chu, P., Saucier, D.A. \& Hafner, E. (2010). Meta-analysis of the relationships between social support and weII-being in children and adolescents. Journal of Social and Clinical Psychology, 29 (6), 624-645 DOI: 10.1521/jscp.2010.29.6.624

Cobb, S. (1976). Social support as a moderator of life stress. Psychosomatic Medicine, 38, 300-314.

Cohen, S. \& Willis, T. A. (1985). Stress, social support and the buffering hyphotesis, Psychological Bulletin, 98, 310-357.

Conger, R. \& Conger, K. (2002). Resilience in midwestern families: selected findings from the first decade of a prospective, longitudinal study. Journal of marriage and family, 64, 361-373.

Coppari, N. (2013) Adolescencia Prevenida: Empoderando en la Promoción de la Resiliencia en Talleres de Formación de Monitores. En J. Gaxiola \& J. Palomar (Coords.). Estudios de resiliencia en América Latina. (Vol. 2). (pp. 121-136) CDMX, México: Pearson, Universidad de Sonora y Universidad Iberoamericana.

De la Caridad M.S., M. (2013). Contextualizando la relación pobreza, salud e inclusión social desde una mirada a la atención primaria de salud como espacio social. Revista Caribeña de Ciencias Sociales. Re- 
cuperado de http://xn--caribea-9za.eumed.net/ wp-content/uploads/pobreza-salud.pdf

Dirección General de Estadísticas, Encuestas y Censos (DGEEC) (2016). Principales resultados Encuesta permanente de hogares. Documento Oficial. Asunción, Paraguay

Fernández P., R. (2005). Redes sociales, apoyo social y salud. Perifèria (3), 1-16. Recuperado de http://revista-redes.rediris.es/Periferia/english/number3/periferia_3_3.pdf

Fresno R., A., Spencer C., R., Leiva B., M. \& Gallardo C., I. (2011). Ingreso familiar y variables psicológicas asociadas a la pobreza como predictores de la calidad de la representación del apego en niños preescolares en Chile. Salud \& Sociedad, 2 (2), 176-192. DOI: http:// dx.doi.org/10.22199/S07187475.2011.0002.00004

Hamui-Sutton, A., Ponce-Rosas R., E., Irigoyen-Coria, A. \& Halabe-Cherem, J. (2009). Capital social, pobreza familiar y autopercepción de apoyo en casos de enfermedad respiratoria aguda. Gaceta Médica de México, 145 (6), 491-500. Recuperado de https:// www.researchgate.net/publication/267986056_ Capital_social_pobreza_familiar_y_autopercepcion_de_apoyo_en_casos_de_enfermedad_respiratoria_aguda

Hickey, E., Fitzgerald, A. \& Dooley, B. (2017). The relationship between perceived family support and depressive symptoms in adolescence: what is the moderating role of coping strategies and gender? Community Mental Health Journal, 53, 474-481 DOI: 10.1007/ s10597-017-0087-x

Kaniasty K. \& Norris F. H. (2001). Social support dynamics in adjustment to disasters. En Sarason B. R., Duck S. (Eds.), Personal relationships: Implications for clinical and community psychology (pp. 201-224). New York, USA: Wiley \& Sons.

Marmot, M. (2015). The health gap: the challenge of an unequal world. The Lancet, 386 (10011), 2442 - 2444. DOI: http://dx.doi.org/10.1016/S0140-6736(15)00150-6

Martín, E., Fajardo, C., Gutiérrez, A. \& Palma, D. (2007). Estrategias de afrontamiento de crisis causadas por el desempleo en familias con hijos adolescentes en Bogotá. Acta colombiana de Psicología, 10(2), 127-141.

Martínez, A. (2004). Evaluación de la confiabilidad de dos escalas de apoyo social: la escala de apreciación social y la escala de apoyo social. (Tesis inédita de licenciatura). FES Iztacala. UNAM, México.

McGuigan, F. J. (1996) Psicología Experimental. CDMX, México: Trillas.

Martos, M. J. \& Pozo, C. (2011). Apoyo funcional vs. disfuncional en una muestra de pacientes crónicos: Su incidencia sobre la salud y el cumplimiento terapéutico. Anales de Psicología, 27, 47-57.

Méndez, P. \& Barra, E. (2008). Apoyo Social Percibido en Adolescentes Infractores de Ley y no Infractores. Psykhe (Santiago), 17(1), 59-64. https://dx.doi. org/10.4067/S0718-22282008000100006

Matud A., M.P., Correa R., M.C. \& García P., L. (2017). Relevancia de la menopausia y del apoyo social en las diferencias en salud entre mujeres y hombres, Universitas Psychologica, 16(1), 1-11. http://dx.doi. org/10.11144/J averiana.upsy16-1.rmas

Montesinos, R., Lloret, D., Segura, J.V. \& Aracil, A. (2016). La percepción del apoyo social en jugadores patológicos y su relación con el éxito del tratamiento. Clínica y Salud, 27, 15-22. Recuperado de http:// eds.b.ebscohost.com/eds/pdfviewer/pdfviewer?$\mathrm{vid}=0 \& \mathrm{sid}=33 \mathrm{aa} 8 \mathrm{e} 38-2 \mathrm{a} 67-48 \mathrm{~b} 6-9 \mathrm{f} 8 \mathrm{a}-\mathrm{dfd} 0 \mathrm{cb}-$ 74d6e0\%40sessionmgr101.

Musitu, G. \& Cava, M.J. (2003). El rol del apoyo social en el ajuste de los adolescentes. Psychosocial Intervention, 12(2) 179-192. Recuperado de http://www.redalyc.org/articulo.oa?id=179818034005.

Office for National Statistics (2014). Inequality in healthy life expectancy at birth by national deciles of area deprivation: England [en línea]. Statistical Bulletin. Recuperado de http://www.ons.gov.uk/ons/ rel/disability-and-health-measurement/inequality-in-healthy-life-expectancy-at-birth-by-national-deciles-of-areadeprivation-england/2009-11/ index.html

Palomar L., J. \& Cienfuegos M., Y. I. (2007). Poverty and social support: a comparative study in three socioeconomic levels. Interamerican Journal of Psychology, 41(2), 177-188. Recuperado de http:// pepsic.bvsalud.org/scielo.php?script=sci_arttext\&pid $=$ S0034-96902007000200008\&lng =en\&tl$\mathrm{ng}=\mathrm{en}$.

Ramírez, A. (2013). Interacción del sexo, la edad y el tipo de familia en la percepción del apoyo social en adolescentes. (Tesis inédita de licenciatura). Facultad de estudios superiores Zaragosa, UNAM, México.

Ramírez, A. (2015). Percepción de apoyo social en adolescentes de contextos múltiples. En B. Barcelata, Adolescentes en riesgo. Una mirada a partir de la resiliencia (pp. 85 - 108) México, CDMX: Manual Moderno.

Ramos-Díaz, E., Rodríguez-Fernández, A., Fernández-Zabala, A., Revuelta, L., \& Zuazagoitia, A. (2016). Apoyo social percibido, autoconcepto e implicación escolar de estudiantes adolescentes. Revista de Psicodidáctica, 21(2), 339-35. DOI: 10.1387/RevPsicodidact.14848

Rodríguez E., S. (2010). Relación entre nivel socioeconómico, apoyo social percibido, género y depresión en niños. Interdisciplinaria, 27(2), 261-276. Recuperado de http://www.scielo.org.ar/scielo.php?script=sci_arttext\&pid=S1668-70272010000200005\&ln$\mathrm{g}=$ en\&tlng=en.

Rodríguez-Fernández, A., Ramos-Díaz, Eı, Ros, I., Fernández-Zabala, A. \& Revuelta, L. (2016). Bienestar subjetivo en la adolescencia: el papel de la resiliencia, el autoconcepto y el apoyo social percibido. Summa Psicológica, 23(1), 60-69 DOI: https:// doi.org/10.1016/j.sumpsi.2016.02.002

Rodríguez-Fernández, A.; Ramos-Díaz, E.; Ros, l. \& Zuazagoitia, A. (2018). Implicación escolar de estudiantes de secundaria: La influencia de la resiliencia, el autoconcepto y el apoyo social percibido. Educación XX1, 21(1), 87-108, DOI: 10.5944/educXX1.16026

Rosenblüth, M. (2006). Una propuesta para actualizar la canasta de satisfacción de necesidades básicas. En R. Camhi \& R. Castro (Eds.) La nueva realidad de la pobreza en Chile, (pp. 169-216) Santiago de Chile, Chile: Fundación Libertad Desarrollo.

Rutter, M. (2002). Psychosocial resilience and protective mechanisms. En J. Rolf, A. Masten, D. Ciccheti, K. 
Nuechterlein \& Sh. Weintraub. Risk and protective factors in the development of psychopatology ( $\mathrm{pp}$. 181-214), New York, USA: Cambridge University Press.

Salazar C., R., Pereira D.B., T. R., Silveira V., A., \& Lost P., S. C. (2013). Social support in the context of poverty: a study of the elderly with cognitive impairments and their family caregivers. Revista de Pesquisa: Cuidado e Fundamental, 5(2), 3787. DOI:10.9789/21755361.2013v5n2p3787

Teherán V., A.A., Mejía G., M.C., Álvarez M., L.J., Muñoz R., Y.J., Barrera C., M.C. y Cadavid G., V. (2017). Relación entre el apoyo social y las complicaciones agudas de la diabetes tipo 2: un estudio de corte transversal. Ciencias de la Salud, 15(2), 211-222. http:// dx.doi. org/10.12804/revistas.urosario.edu.co/revsalud/a.5757

UNICEF (2005). Desafíos: Boletín de la infancia y adolescencia sobre el avance de los objetivos de desarrollo del milenio. Santiago, Chile: CEPAL, UNICEF. Recuperado de http://hdl.handle.net/11362/35975

Vakis, R., Rigolini, J. \& Lucchetti, L. (2015). Los olvidados: pobreza crónica en América Latina y el Caribe. Resumen ejecutivo. Recuperado de http://agris.fao.org/ agris-search/search.do?recordID=US2015601183

Werner, E. \& Smith, R. (1992). Overcoming the odds. New York, USA: Cornell University Press 